\title{
Further research on the coupled Influence of Temperature and Stress Field to PCB' Modal
}

\author{
Qin Luo ${ }^{1, a,{ }^{*}}$, Ke Yao ${ }^{1,2, b}$ and Chuangmian Huang ${ }^{1,3, c}$ \\ ${ }^{1}$ The Fifth Electronics Research Institute of Ministry of Industry and Information Technology, \\ Guangzhou, 510610, China \\ ${ }^{2}$ Guangdong Provincial Key Laboratory of Electronic Information Products Reliability Technology, \\ Guangzhou 510610, China \\ ${ }^{3}$ Guangdong Provincial Engineering Laboratory for reliability of industrial robot, Guangzhou 510610, \\ China \\ aluoqin5492143@sina.com, byaok_@126.com, chuangchuangmian@163.com
}

\begin{abstract}
The coupling analysis of temperature and vibration has been an important branch of domestic and international research. Influence of Temperature and Stress Field to PCB' Modal has been described in previous articles ${ }^{[1]}$. In this paper, vibration stress analysis method based on ANSYS considering temperature field and thermal stress field is introduced. Further research on the coupled influence of temperature and stress field to PCB' modal is analyzed. And the temperature field, stress field and the coupled Influence on PCB's modal were compared, which provide a reference for the PCB design and modal analysis in the future.
\end{abstract}

Keywords: coupled influence, temperature field, stress field, PCB’s modal.

\section{Introduction}

Electronic components are the key components of the weapon equipment and electronic equipment. It is usually affected by the temperature and vibration in the service process. Studies have indicated that temperature and vibration are the main reasons for the failure of electronic equipment, and the coupling of temperature and vibration usually accelerates the failure of electronic equipment.

Studies have shown that the dynamic effects of transient temperature and stress field coupled mainly to the impact of temperature and stress fields on the structural stiffness ${ }^{[1]}$. The increasing of temperature leads the diversification of materials physical properties and causes a marked reduction in mechanical property ${ }^{[2]}$. On the other side, the inhomogeneous temperature field and temperature gradient forms in the structure. The caused thermal stress will also change the rigidity of structure and the rigidity distribution, leading to the dropping of torsional rigidity and flexural rigidity of structure ${ }^{[3]}$.

In this paper, a PCB model was created to calculate the temperature field and the stress field base on the transient thermal analysis and thermal-structure coupling analysis. The coupled Influence of Temperature and Stress Field to PCB' Modal is analyzed, by using the iteration of PCB's thermal modal analysis. And the Influence of temperature field, stress field and the coupled on PCB's modal were compared, which provide a reference for the PCB design and modal analysis in the future.

\section{Analysis of modal considering temperature field and thermal stress field}

Process of vibration stress considering temperature field and thermal stress field is divided into three stages: Finite element analysis model, thermal modal analysis, and random vibration Analysis. Flow chart of Analysis of vibration stress considering temperature field and thermal stress field as shown in figure 1. 


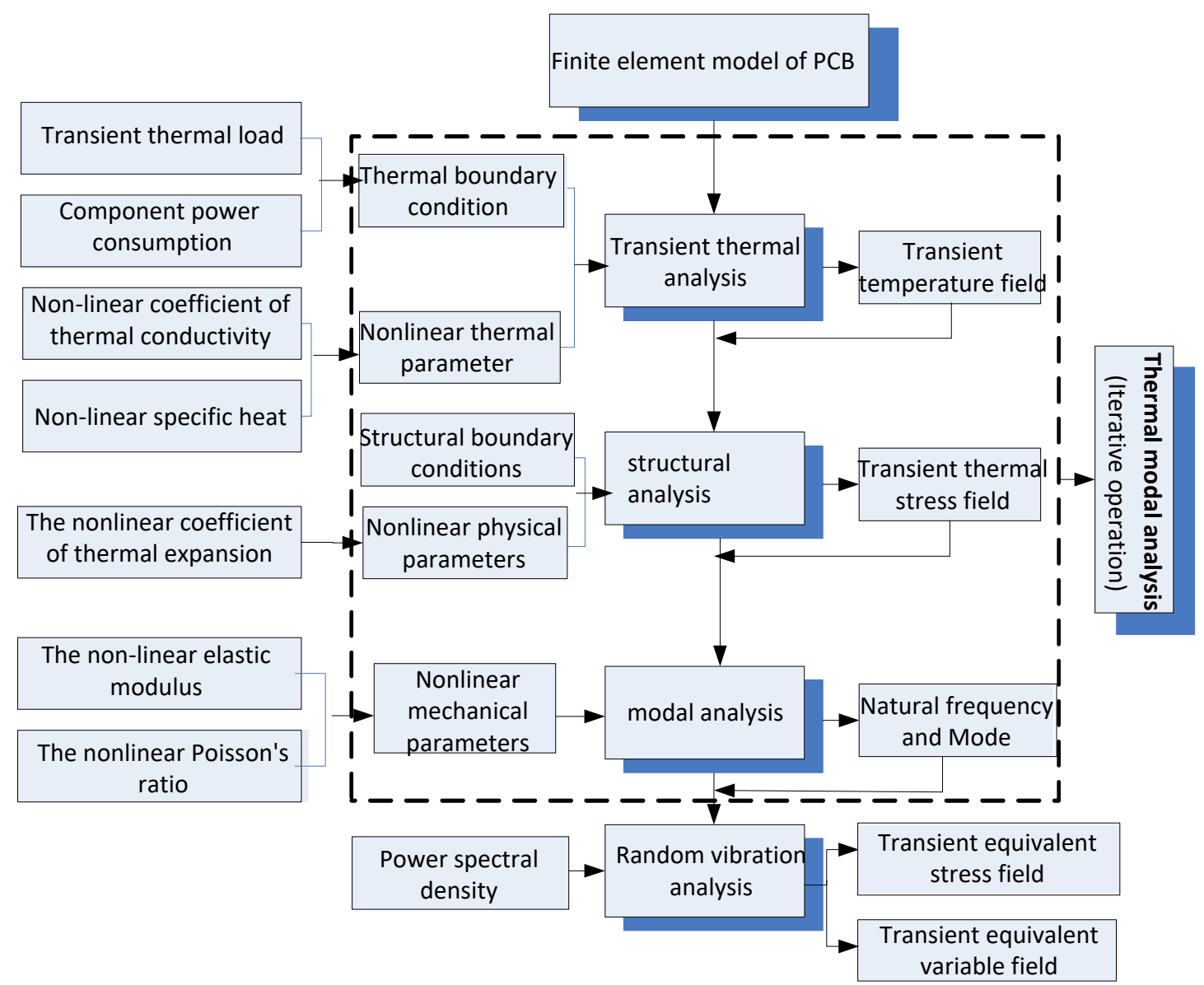

Fig. 1 Vibration stress analysis method based on ANSYS considering temperature field and thermal stress field

Thermal modal analysis, considering the effects of temperature and thermal stress field, is based on thermal analysis and structural analysis. PCB's thermal modal analysis process is divided into three stages: transient thermal analysis, thermal structural analysis, and modal analysis.

Taking into account the effect of the material non-linearity on the mechanical properties of the PCB, the material parameters of the PCB should be set different material parameter values for changing temperatures during the analysis process. As the component contains a variety of materials, each material parameter changes with temperature are different, and it has less effect on board-level circuit parameters on the dynamic characteristics, the material parameters of components considered for the average constant. PCB's modulus, the relationships of Poisson's ratio, thermal expansion coefficient, specific heat with temperature can be seen in literature ${ }^{[4]}$.

The influence of temperature on modal, mainly due to temperature effects on the structural stiffness, and thermal stress will also change the rigidity of structure and the rigidity distribution, leading to the dropping of torsional rigidity and flexural rigidity of structure ${ }^{[3]}$. Therefore:

- if considering the effect of stress field on the PCB's modal, set thermal structural analysis results as Modal loads, but the structure is uniform temperature, when calculating the modal;

- If considering the temperature effect on material properties, load temperature field, while the internal structure of the initial stress-free, when calculating the modal;

- If considering the coupled influence of temperature and stress Field on the PCB's modal, set thermal structural analysis results as prestress load and load temperature field as thermal load , when calculating the modal. 


\section{Case application}

The PCB is an irregular shape as Shown in Fig.1. The initial temperature is set to be $25^{\circ} \mathrm{C}$. In the case of natural convection, the film coefficients of the PCB and the packages are $6.0 \times 10-6$ and $1.0 \times 10-5$ $(\mathrm{W} / \mathrm{mm} 2 \cdot \mathrm{K})$.

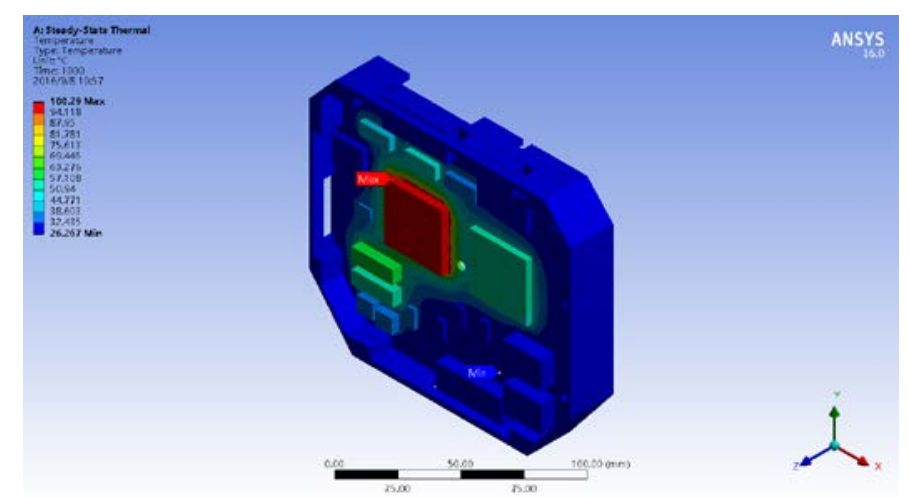

Fig.1 PCB model

Through the transient analysis, it is known that the temperature of PCB can reach stable after 4000 seconds due to the power consumption of the components. That is to say, the temperature of PCB has been changed in the 400 seconds, and the change of the temperature field has caused the change of the stress field. The result of modal considering temperature field and thermal stress field are shown in fig.2.
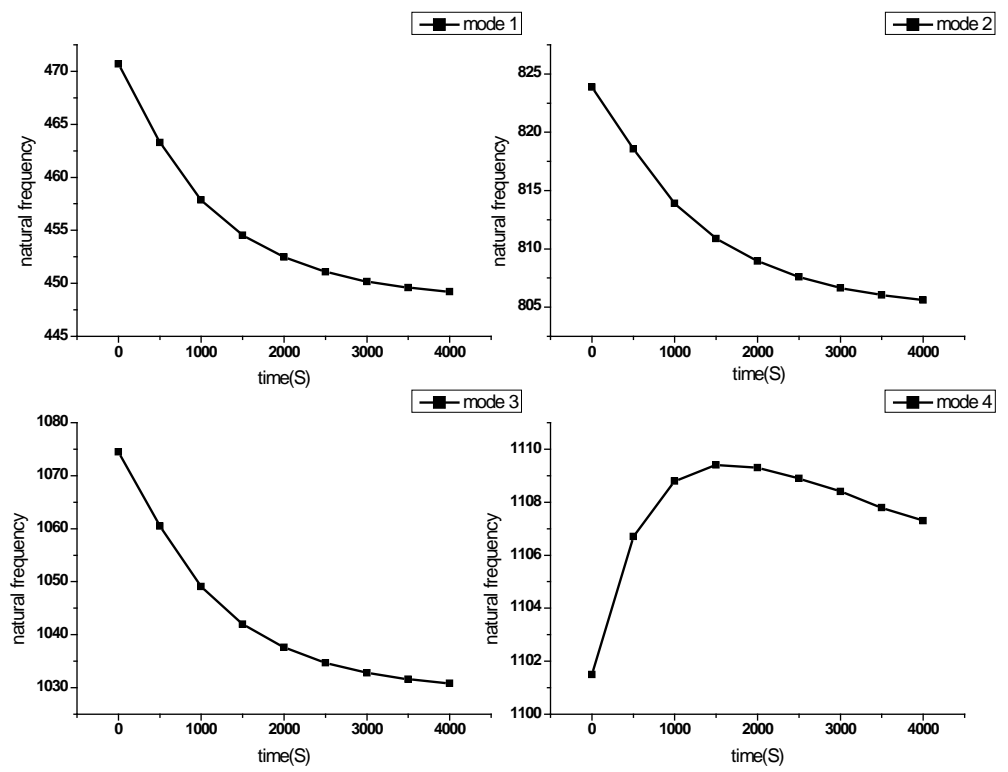

Fig. 2 The result of modal considering temperature field and thermal stress field

In 4000 seconds, the first 4 natural frequencies of PCB decreased by $4.57 \%, 2.22 \%, 4.07 \%$ and $-0.53 \%$.This change is caused by the power consumption of the components in the case of constant ambient temperature.

\section{Comparative analysis}

The comparison of the effects of the temperature field, stress field and the coupled on the modal is shown in fig3. As can be seen from the figure, the first three orders of natural frequency with the increase of time have a downward trend.

The temperature field makes the fourth order natural frequency of PCB increased and then decreased. This is due to the first 2000 seconds, PCB temperature changes very fast, so the thermal stress caused by the relatively large, and with the increase of time PCB temperature field gradually reached a stable, 
thermal stress gradually. Finally, the thermal stress field is the dominant factor, which makes the natural frequency of PCB first increased and then decreased.
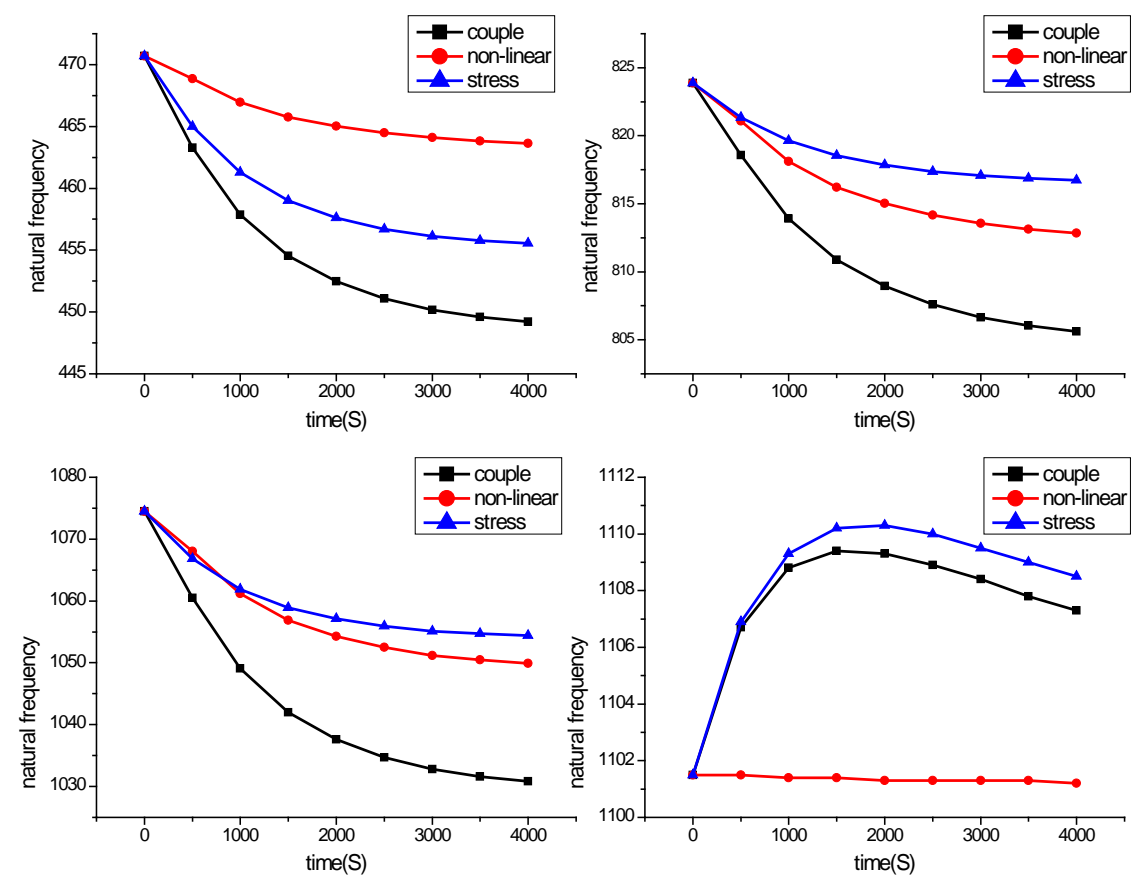

Fig.3 the comparison of the effects of the temperature field, stress field and the coupled on the modal

\section{Conclusions}

In this paper, vibration stress analysis method based on ANSYS considering temperature field and thermal stress field is introduced. The influence of temperature field and thermal stress field on the vibration stress is realized by the iteration of PCB's transient thermal structure analysis and thermal modal analysis.

Vibration stress analysis method based on ANSYS considering temperature field and thermal stress field is introduced. Further research on the coupled influence of temperature and stress field to PCB' modal is analyzed. In 4000 seconds, the first 4 natural frequencies of PCB decreased by $4.57 \%$, $2.22 \%, 4.07 \%$ and $-0.53 \%$.

The temperature field, stress field and the coupled Influence on PCB's modal were compared. The first three orders of natural frequency with the increase of time have a downward trend. The fourth order natural frequency of PCB increased and then decreased. This is due to the first 2000 seconds, PCB temperature changes very fast, so the thermal stress caused by the relatively large, and with the increase of time PCB temperature field gradually reached a stable, thermal stress gradually. Finally, the thermal stress field is the dominant factor, which makes the natural frequency of PCB first increased and then decreased.

\section{References}

[1] Qin Luo, Sujuan Zhang and Xiao Zhang. Study on the Influence of Temperature and Stress Field to PCB’s Modal, J. applied mechanics \& materials. 2013, 271-272:1441-1445.

[2] JianQinglong, Hu Zhigang. Finite Element Analysis for Temperature-stress Coupled Field of the PCB, J. Electronic Product Reliability and Environmental Testing. 2009(1):39-42.

[3] JI Kexing, Song Hongwei, Huang Chenguang. Influence of Transient Temperature and Thermal Stress on the Vibration Mode of Active Cooling Scramjet, C. Third Hypersonic Technology Conference, 2010. 
[4] Zhu Ji-yuan, Zhou De-Jian. Study and Analysis of Thermal-vibration Coupling Characteristics of Board Level Circuit Modules, J. Electro-mechanical Engineering. 2007, 23(6):4-7. 